\title{
META-ANALYSIS OF QUALITY OF LIFE OUTCOMES FOLLOWING DIABETES SELF-MANAGEMENT TRAINING
}

\author{
Jane Cochran, PhD, RN, CDE and Vicki S. Conn, PhD, RN, FAAN \\ Jane Cochran: ; Vicki S. Conn: conn@missouri.edu
}

\begin{abstract}
Purpose-The purpose of this meta-analysis was to meet the need to quantify the influence of diabetes self-management training on quality of life (QOL) of adult diabetes patients.

Methods-Extensive literature searching located published and unpublished diabetes selfmanagement intervention studies that measured QOL outcomes among at least 5 subjects with type 1 or 2 diabetes. Data were extracted from primary study reports which included interventions designed to improve diabetes self-management and adequate data to calculate effect sizes. Randomeffects meta-analytic procedures were used to estimate overall effects between treatment and control groups at outcome assessment and between baseline and outcome data for both treatment subjects and control subjects.
\end{abstract}

Results-Exhaustive searching yielded 20 comparisons across 1,892 subjects. The comparisons between treatment and control group outcomes following interventions yielded an effect size of 0.281 . The comparisons between treatment group at baseline and outcome measurement yielded an effect size of 0.312 to 0.313 . Each of these effect sizes were statistically significant, meaning that the hypothesis that interventions to improve diabetes self-management results in increased QOL was supported. Control subjects did not experience improved QOL while participating in studies.

Conclusion-These findings document that people with diabetes experience improved QOL from participation in diabetes self-management training programs. Future diabetes self-management intervention studies should include quality of life outcomes so that this important outcome can be further studied. After more primary studies are available future meta-analyses can explore important moderator analyses.

Researchers and clinicians are increasingly considering the influence of diabetes care burden and complications on quality of life (QOL). QOL has been found to be lower in persons with diabetes when compared to individuals that do not have diabetes. ${ }^{1,2}$ Among adults with diabetes, QOL decreases over time despite improvements in clinical outcomes. ${ }^{3}$ The importance of QOL has been recognized as an important outcome of diabetes care as well as a factor in sustaining the ongoing performance of diabetes self-care activities. ${ }^{4}$

While QOL in diabetes patients has been studied in relation to patient demographics, type of diabetes and diabetes treatment, it historically has not routinely measured as part of diabetes self-management training clinical trials. Glasgow found that only $17 \%$ of diabetes selfmanagement training clinical trials reported in the late 1990's included a QOL outcome. ${ }^{5}$ With the increased attention to QOL outcomes, researchers have begun reporting this important outcome in intervention trials.

Correspondence to: Vicki S. Conn, conn@missouri.edu.

The final version of this paper has been published in The Diabetes Education, 34, 815-823, 2008 by SAGE Publications Ltd, All rights reserved. It is available at: http://TDE.sagepub.com/ 
No previous meta-analyses have addressed QOL outcomes after diabetes self-management interventions. As part of their 2001 meta-analysis of the effects of diabetes education, Norris, Engelgau and Narayan found only three studies that had QOL as an as outcome. Two of these found no change in QOL with the third showing an improvement in QOL. They did not synthesize across this small number of studies. ${ }^{6}$ A meta-analysis of psychologically-focused interventions in diabetes patients found a slight decrease in psychological distress following the interventions. ${ }^{7}$ In a review of randomized controlled trials of diabetes educational interventions between 2001 and 2005, eight studies found improvements in well-being, anxiety and attitude towards diabetes after the intervention. ${ }^{8}$ None of the previous reviews have conducted a quantitative synthesis using meta-analysis. The purpose of this study was to meet the need to quantify the effects of diabetes self-management training on QOL outcomes in adult diabetes patients. The research question for this meta-analysis was: Does diabetes selfmanagement training affect the reported QOL of adult diabetes patients?

\section{METHODS}

This project was performed using meta-analysis methods. Meta-analysis is the systematic integration of primary studies with statistical analysis of their results in order to calculate a pooled effect size for the dependent variables under study. It also allows for further examination of the effect of moderators on the dependent variable across studies. ${ }^{9}$ Meta-analysis can also be helpful in integrating the results from primary studies that may have had inadequate sample sizes in order to have sufficient power to establish significance. ${ }^{10}$

\section{Inclusion Criteria and Search Strategies}

This project utilized data from a larger meta-analysis that examined the effects of interventions designed to increase physical activity in adults with chronic illnesses. The parent study retrieved any intervention study that included a recommendation to increase physical activity, including diabetes self-management interventions. Further details of the meta-analysis methods used in the parent study are available elsewhere. ${ }^{11-13}$ A subset of the parent study primary studies that examined diabetes self-management training in adults with diabetes with reported QOL outcomes was analyzed for this project. Primary studies were included in this analysis if they were coded as having adult participants with diabetes, a diabetes selfmanagement training intervention, and a QOL outcome.

An extensive literature search was performed as part of the parent study identifying primary studies from 1970 to April 2005 that reported outcomes following a diabetes self-management training intervention. The literature search included published and unpublished studies to avoid publication bias. Examples of unpublished studies include dissertations, conference abstracts and unpublished manuscripts. Publication bias may over estimate the total effect size because negative results or results that failed to reach statistical significance are often not published. 14,15

The parent project examined multiple electronic databases to ensure a comprehensive search. 16 A reference librarian performed an electronic search of 11 databases including CINAHL, Clinical Evidence, Cochrane Central Register of Controlled Trials, Dissertation Abstracts, EMBASE, ERIC, HealthStar, MEDLINE, PsychInfo, and SportDiscus. Table 1 lists the terms used for the literature search in the parent project. Hand searches were performed by the research team in the journals Diabetes, Diabetes Care, The Diabetes Educator, Diabetes Medicine, Diabetes Research and Clinical Practice, Diabetes Spectrum and Diabetologia. Conference abstracts from the American Diabetes Association and the American College of Sports Medicine were explored. 
In an effort to be comprehensive, ancestry searches of the identified primary studies, narrative reviews and syntheses of the topic were done. Searches of National Institutes of Health funded study register were also performed. Additionally senior authors of primary studies were contacted and computerized searches of senior authors were conducted. ${ }^{17}, 18$ Comprehensive searching in the parent project yielded 2807 papers, 127 with HbA1c outcomes and 35 with fitness outcomes. Most paper were excluded for not reporting on an intervention study or not reporting codeable outcome data.

For this project, studies were included if they reported data from instruments which measured QOL (e.g. health-related QOL, life satisfaction, well-being, or psychosocial adaptation to illness) as outcomes from self-management interventions. These terms were chosen because of their frequency of use as synonyms of QOL in the primary studies. We did not limit the synthesis to a specific QOL measure because no such measure has universal acceptance. Furthermore, when constructs are measured imprecisely, such as QOL, including some diversity of measures is helpful. The identification and measurement of mood, anxiety and depression were not coded as QOL outcomes. Although mood may be related to QOL, it is not an adequate proxy measure of QOL. Thus we focused on the homogeneous construct of quality of life assessed with heterogeneous measures.

Studies with participants with either type 1, type 2 or unspecified type of diabetes were included. The decision to include both types of diabetes was based on the nature of the interventions and outcome variables. We included both types of diabetes for three reasons. First, there are few systematic differences between diabetes self-management classes for adults with type 1 and type 2 diabetes. Second, the recommended self-management behaviors are similar for both types of diabetes. Finally, there is little reason to believe the effects of intervention to improve self-management behavior on QOL would differ between types 1 and 2 diabetes. (Our previous work on metabolic control and fitness outcomes did not combine types 1 and 2 diabetes because these outcomes likely differ significantly between diabetes types.) Further inclusion criteria used for the primary studies are in Table 2.

Both two-group and single-group comparison primary studies were included. Two group studies compare treatment and control subjects following the intervention. Single group studies only compare pre- and post-intervention data from the same group of subjects. Baseline and outcome data were coded for both treatment and control subjects, and these were analyzed these as single-group data even among studies with both treatment and control groups. The inclusion of studies was not limited to a particular research design, to capture the broad spectrum of completed primary research. ${ }^{10}$

\section{Data Extraction}

The primary reports were coded using a coding frame that had been developed for the parent project, then piloted and revised accordingly. To enhance coding reliability, each primary study was independently coded by two coders with the principal investigator of the larger metaanalysis resolving any discrepancies between coders.

Primary studies were coded for year of publication, study type, participant age, type of diabetes, gender, body mass index (BMI), ethnicity, glycemic control pre- and/or post-intervention, intervention duration, group versus individual intervention, characteristics of exercise prescription, exercise as only behavioral target or multiple diabetes self-management behavior targets, intervention components, fitness level, QOL pre- and/or post-intervention, time between intervention and outcome measures, and type of QOL measurement tool. 


\section{Analysis}

The standardized mean difference effect size selected for this project is consistent with primary research where QOL mean scores are typically reported. The effect size was determined by calculating the standardized mean difference for each comparison between treatment and control groups or pre- and post-intervention results for single group design studies. The effect size reflects the difference between the treatment versus control group outcome scores (standardized by dividing the difference by the pooled standard deviation) for two group comparisons. For single-group studies, the effect size depicts the difference between the treatment group after the intervention compared to the same group's scores prior to the interventions, divided by the pooled standard deviation.

To account for differing sample sizes in the primary studies, the effect size's of the individual studies were weighted by the inverse of their variance thus giving larger sample primary studies more influence on overall QOL effect size values. The effect size demonstrates the impact the intervention had on the QOL outcomes. A positive treatment effect on QOL is reflected by a positive effect size score. The effect size for each primary study was calculated and then these effect sizes were combined to obtain an overall effect size. This calculation of effect size reflects comparable mean results regardless of the QOL outcome measure used. Pooling different QOL measures for determining effect size in meta-analyses has been supported in the literature although caution should be used depending on the constructs being tested and responsiveness of instruments. ${ }^{19}$

Studies using two-group comparisons were analyzed separately from studies with a singlegroup design. Studies with multiple treatment groups but no control group were included with the single-group studies. Analysis of single-group studies can be problematic since the preand post-test results are most likely correlated. Unfortunately, none of the primary studies reported this information; therefore the analyses were conducted using the assumption of no correlation as well as the assumption of a strong positive correlation between pre- and posttest scores. Both data are presented.

A random effects model was used in the analysis. The random-effects model assumes that there are sampling errors as well as study implementation variances that may affect the effect sizes of the primary studies. Use of a random-effects model is appropriate for this meta-analysis because the implementation of self-management training is heterogeneous. The random effects model allows for increased generalization of the findings to other studies with differing characteristics. ${ }^{20,21}$ The between study variance was computed by the weighted method of moments. A conventional heterogeneity statistic $(Q)$ was calculated to determine effect size homogeneity between primary studies.

A formal analysis of potential moderator effects on QOL was not performed because potential moderating factor information was insufficiently reported in the primary studies. Primary study source, participant and intervention data were therefore only tabulated.

\section{RESULTS}

Extensive searching yielded 2,807 citations for possible inclusion. Abstracts and full papers were evaluated for the inclusion criteria, which dramatically narrowed the potential studies. Papers were commonly excluded because the study lacked either an intervention or a QOL measure. The final yield was 20 comparisons across 1,892 subjects that were eligible for inclusion. ${ }^{22-37}$ The earliest study was published in 1991, while most of the studies $(k=13)$ were published in 2000 or later ( $k$ denotes the number of comparisons). Nineteen studies were published; one was a dissertation. Two studies were not funded; the remainder received some source of funding to conduct the study. 


\section{Samples included in Primary Studies}

Descriptive information about the studies is provided in Table 3. Most samples were middleaged and older. Only 4 studies reported a mean age under 57 years. Women were well represented in these samples. Only 2 studies excluded women. Only 6 studies reported including African-Americans. Among these 6 studies, 3 studies focused exclusively on African Americans. None of the studies that included African Americans and other subjects reported data separately for African Americans. No studies reported including Latino or Native American subjects.

Most studies contained many overweight subjects (mean BMI $=33.34$ ). Only 2 of the 7 studies that reported BMI reported means less than 30 . Metabolic control on entry into studies was poor (mean $\mathrm{HbAlc}=8.74$ ), with 4 studies reported entry HbA1c over 10 . Too few studies reported $\mathrm{HbA1c}$ levels after the interventions to analyze outcomes. Sixteen studies focused on adults with type 2 diabetes, 3 included only type 1 diabetic subjects, and 1 study included both types of diabetes. Inclusion of co-morbidity, education or socioeconomic information was too inconsistently reported for analysis.

\section{Interventions}

Interventions varied greatly (Table 3 ). Two studies delivered interventions in 15 minute sessions and 4 studies used sessions of at least 3 hours. Four studies used more than 10 intervention sessions. Over half of the interventions were delivered in 4 months or fewer. One study delivered the intervention over 1 year and 1 study spread the intervention over 4 years. Only 3 studies reported theoretical frameworks for interventions (transtheoretical model, cognitive behavioral theory, behavior modification theory). In addition to diabetes selfmanagement education, the most frequent intervention components included barriers management (7), problem solving (7), goal setting (5), social support (5), social modeling (4), relapse prevention education (2), and supervised exercise (2). Intervention strategies reported by only 1 study included competition, rewards, decision making, exercise prescription, fitness testing, and self-reevaluation. Two interventions were individually tailored for subjects. Over half of the intervention sessions used discussion format as well as didactic lecture. Six studies delivered at least part of the intervention via telephone and 1 study used mail delivery. Only 2 studies did not include any face-to-face delivery of interventions. Among the studies that reported the physical setting where interventions were delivered, 9 were in ambulatory care settings, 4 were in community centers, and 1 was delivered to in-patients. Seven studies presented interventions to individual subjects while 13 used groups. Most of the 17 studies that described interventionist professional characteristics reported combinations of health professionals as interventionists $(k=10)$.

\section{Methodological Characteristics}

Small samples were common (Table 3). Six studies included less than 30 subjects. Eight comparisons could be made between treatment groups and control groups following interventions. Twenty studies provided baseline and outcome data for treatment subjects for making single group pre- versus post-group comparisons. Six of the two-group comparisons used random assignment of subjects to treatment and control groups. Two of the two-group comparisons did not use random assignment. QOL was most often measured by the Medical Outcomes Study Short Form $36(k=6)$. Only 1 study used an investigator developed instrument and while the remainder used previously developed measures of QOL.

\section{QOL Outcomes}

The comparison between treatment group and control group outcomes following interventions yielded an effect size of $0.281\left(\hat{\mu}_{\delta}=0.281\right)$ (Table 4$)$. The comparison between treatment group 
at baseline and outcome measurement yielded an effect size of $0.312\left(\hat{\mu}_{\delta}=0.098\right)$ (Table 4) under the assumption of high correlation between pre- and post-test scores. Results of the analysis with the assumption of no correlation for single-group pre- versus post-intervention comparison was similar with an effect size of $0.313\left(\hat{\mu}_{\delta}=0.110\right)$. Each of these effect sizes were statistically significant, meaning that the hypothesis that interventions to improve selfmanagement results in increased QOL outcomes was supported.

In contrast, neither of the effect sizes for control group pre- versus post-intervention QOL scores were significantly different from 0 (Table 4). Control subjects did not experience improvements in QOL by participating in these studies.

The $Q$ values reported in Table 4 document that the studies were significantly heterogeneous across two-group (72.934) and single-group comparisons (490.271 and 109.546). This was expected because diverse interventions were tested in varied samples using a wide range of methods in these primary studies.

\section{DISCUSSION}

The meta-analysis findings document that people with diabetes experience improved QOL after receiving interventions designed to improve their diabetes self-management behaviors. Previous reviews retrieved too few studies to conduct a meta-analysis. ${ }^{6}$ Two previous syntheses of small numbers of primary studies found decreases in psychological distress and symptoms, but did not directly address QOL. ${ }^{7}, 8$ This is the first meta-analysis to synthesize QOL outcomes in adult diabetes patients following diabetes self-management training. These findings are important because health care is increasingly focused not only on decreasing morbidity, mortality, and costs but also on increasing QOL especially among those with major chronic illnesses such as diabetes.

The magnitude of the effect size is difficult to assess because too few studies used any single measure in exactly the same way to allow us to convert the effect size to an original metric. Although .28 effect size may appear small, a recent meta-analysis of HbA1c outcomes reported an effect size of .29 which equated to post-intervention HbA1c of 7.38 for treatment subjects as compared to 7.83 for control subjects. ${ }^{11}$ Thus what appears to be a small effect size may be clinically meaningful. As more studies use identical measures of QOL, the amount of change required to be clinically meaningful may become clearer. Since QOL is a complex phenomena likely affected by diverse factors, any statistically significant improvement is probably clinically important.

The reasons for this QOL improvement are not known since the interventions were not designed to affect QOL. It is possible that people may feel better with improved metabolic control following interventions. This link was not able to be explored through moderator analyses because too few studies reported both QOL and HbA1c outcomes.

One possible explanation for the improvement in QOL involves self-efficacy. Recently there has been a movement away from didactic teaching alone to a focus on attitudes, coping and self-efficacy. ${ }^{38}$ Extant research reported that self-management education increases selfefficacy. ${ }^{39}$ Increased diabetes care self-efficacy has consistently been linked to increased QOL in patients with type 1 and type 2 diabetes. ${ }^{4,} 40$ Thus the influence of self-management interventions on QOL may be mediated by self-efficacy. Another possible explanation is that people may experience improved QOL when they have an enhanced sense of mastery over the potential consequences of diabetes, which may result from better education.

An alternative explanation is that people may experience improved QOL as a result of changing their diet and exercise behavior. Previous research has documented improved QOL following 
positive health behavior change among adults with diabetes. ${ }^{41}$ It is also plausible that subjects experience group camaraderie or social support during the group diabetes classes that result in better QOL outcomes. Future research needs to address these issues.

These findings may be particularly useful in practice settings. Dramatic changes in diet and exercise may feel overwhelming to diabetes patients confronted with the need for life long behavior change. People with diabetes may avoid making these behavior changes because they fear their QOL will be adversely affected. This study's findings suggest the opposite is true. Providers can tell patients with diabetes that evidence suggests people who attend diabetes self-management programs experience improved QOL. Reassurance that they are likely to experience improvements in QOL could be motivating to patients to participate in diabetes self-management programs.

\section{Limitations}

Limitations of this meta-analysis include lack of adequate minority representation in the primary studies' samples. Very few of the primary studies included African Americans and no studies noted inclusion of Hispanic or Native Americans. This relative lack of ethnic inclusion was surprising because of the disproportionate burden diabetes has in these populations.

The parent project for this study included studies with either exercise or $\mathrm{HbA} 1 \mathrm{c}$ outcomes. Far too few of the studies with other diabetes outcomes measured QOL. Future research should include measures of QOL since widely available measures have documented validity and add little respondent burden. Assessing patients' QOL has numerous benefits. It allows health care providers and researchers to better understand what aspects of the illness and treatment the patient views as having the greatest impact on their QOL. It may also be found that the QOL outcome of a particular therapy outweighs its potential benefits. QOL effects of various diabetes treatments may impact patients sustaining health care activities and health care providers decision-making. Understanding QOL may also be useful in communicating to future patients about expectations of the impact treatment has on QOL. ${ }^{42}$

This study shares the limitations of all meta-analyses. The findings are not causal because all meta-analysis findings are observational. As expected, we retrieved a heterogeneous set of studies. This was expected because the interventions used in practice and research vary widely, as do subjects included in samples. After more primary studies become available, heterogeneity may be explored through moderator analyses. This meta-analysis was limited by the number of studies that provided QOL outcome data despite extensive comprehensive searching. We retrieved too few studies to conduct moderator analyses. After more primary studies with QOL outcomes accrue, moderator analyses can determine the impact other variables on QOL, such as sample characteristics like age, ethnicity, gender, type of diabetes, and type of diabetes treatment; intervention attributes such as content and dose; and other outcomes such as the extent to which subjects actually changed their behavior and HbA1c changes.

\section{Implications}

In conclusion, this meta-analysis documented improvements in QOL outcomes among adults with diabetes following interventions designed to enhance their diabetes self-management. Clinicians may use these finding to encourage patients to participate in diabetes selfmanagement training programs. Researchers and providers are encouraged to include QOL outcome measures when evaluating diabetes self-management programs so future metaanalyses can examine important potential moderators. 


\section{Acknowledgments}

Financial support provided by a pre-doctoral fellowship from the National Institutes of Health (1F31NR08322) to Jane Cochran and from a grant from the National Institutes of Health (R01NR07870) to Vicki Conn.

\section{References}

1. Maddigan SL, Feeny DH, Johnson JA. Health-related quality of life deficits associated with diabetes and comorbidities in a Canadian National Population Health Survey. Quality of Life Research 2005;14 (5):1311-1320. [PubMed: 16047506]

2. Saito I, Inami F, Ikebe T, et al. Impact of diabetes on health-related quality of life in a population study in Japan. Diabetes Research and Clinical Practice 2006;73(1):51-57. [PubMed: 16417943]

3. Hill-Briggs F, Gary TL, Baptiste-Roberts K, Brancati FL. Thirty-six-item short-form outcomes following a randomized controlled trial in type 2 diabetes. Diabetes Care 2005;28(2):443-444. [PubMed: 15677813]

4. Rose M, Fliege H, Hildebrandt M, Schirop T, Klapp BF. The network of psychological variables in patients with diabetes and their importance for quality of life and metabolic control. Diabetes Care 2002;25(1):35-42. [PubMed: 11772898]

5. Glasgow RE. Outcomes for and of diabetes research. The Diabetes Educator 1999;25:74. [PubMed: 10711087]

6. Norris SL, Engelgau MM, Narayan KMV. Effectiveness of self-management training in type 2 diabetes: a systematic review of randomized controlled trials. Diabetes Care 2001;24(3):561-587. [PubMed: 11289485]

7. Ismail K, Winkley K, Rabe-Hesketh S. Systematic review and meta-analysis of randomised controlled trials of psychological interventions to improve glycaemic control in patients with type 2 diabetes. Lancet 2004;363(9421):1589-1597. [PubMed: 15145632]

8. Sigurdadottir A, Jonsditter H, Benediktsson R. Outcomes of educational interventions in type 2 diabetes: WEKA data-mining analysis. Patient Education \& Counseling 2007;67:21-31. [PubMed: 17420109]

9. Conn VS. Meta-analysis research. Journal of Vascular Nursing 2004 Jun;22(2):51-2. [PubMed: 15179418]

10. Conn V, Valentine JC, Cooper HM, Rantz M. Grey literature in meta-analyses. Nursing Research 2003;52:256-261. [PubMed: 12867783]

11. Conn VS, Hafdahl AR, Mehr DR, Lemaster JW, Brown SA, Nielsen PJ. Metabolic effects of interventions to increase exercise in adults with type 2 diabetes. Diabetologia 2007 May;50(5):91321. [PubMed: 17342472]

12. Conn V, Hafdahl AR, Lemaster JW, Ruppar T, Cochran J, Nielsen PJ. Meta-analysis of health behavior change interventions in type 1 diabetes. Amerian Journal of Health Behavior. 2007 In Press.

13. Nielsen PJ, Hafdahl AR, Conn VS, LeMaster JW, Brown SA. Meta-analysis of the effect of exercise interventions on fitness outcomes among adults with type 1 and type 2 diabetes. Diabetes Research and Clinical Practice 2006;74(2):111-120. [PubMed: 16735074]

14. Conn V, Isaramalai S, Rath S, Jantarakupt P, Wadhawan R, Dash Y. Beyond MEDLINE for Literature Searches. Journal of Nursing Scholarship 2003;35(2):177-182. [PubMed: 12854300]

15. Czienskowski, U. Meta-analysis - not just research synthesis. In: Schulze, R.; Holling, H.; Bohning, D., editors. Meta-Analysis New Developments and Applications in Medical and Social Sciences. Canbridge, MA: Hogrefe and Huber; 2003. p. 141-152.

16. Royle PL, Bain L, Waugh NR. Sources of evidence for systematic reviews of interventions in diabetes. Diabetic Medicine 2005;22(10):1386-1393. [PubMed: 16176201]

17. Dickersin, K. Publication bias: recognizing the problem, understanding its origins and scope, and preventing harm. In: Rothstein, H.; Sutton, A.; Borenstein, M., editors. Publication Bias in MetaAnalysis Prevention, Assessment and Adjustments. West Sussex, England: Wiley and Sons Inc; 2005. p. 11-34. 
18. Hopewell, S.; Clarke, M.; Mallet, S. Grey literature and systematic reviews. In: Rothstein, H.; Sutton, A.; Borenstein, M., editors. Publication Bias in Meta-Analysis Prevention, Assessment and Adjustments. West Sussex, England: Wiley and Sons Inc; 2005. p. 49-72.

19. Puhan M, Soesilo I, Guyatt G, Schunemann H. Combining scores from different patient reported outcome measures in meta-analyses: when is it justified? Health \& Quality of Life Outcomes 2006;4 (94)

20. Hartung, J.; Knapp, G. An alternative test procedure for meta-analysis. In: Schulze, R.; Holling, H.; Bohning, D., editors. Meta-Analysis New Developments and Applications in Medical and Social Sciences. Cambridge, MA: Hogrefe \& Huber; 2003. p. 53-70.

21. Sauerbrei, W.; Blettner, M. Issues of traditional reviews and meta-analyses of observational studies in medical research. In: Schulze, R.; Holling, H.; Bohning, D., editors. Meta-Analysis New Developments and Applications in Medical and Social Sciences. Cambridge, MA: Hogrefe and Huber; 2003. p. 78-98.

22. Baran R, Crumlish K, Patterson H, et al. Improving outcomes of community-dwelling older patients with diabetes through pharmacist counseling. American Journal of Health System Pharmacy 1999;56 (15):1535-1539. [PubMed: 10478992]

23. Basa RP, McLeod B. Evaluation of a diabetes specialty centre: structure, process and outcome. Patient Education and Counseling 1995;25(1):23-29. [PubMed: 7603930]

24. de Weerdt I, Visser AP, Kok GJ, de Weerdt O, van der Veen EA. Randomized controlled multicentre evaluation of an education programme for insulin-treated diabetic patients: effects on metabolic control, quality of life, and costs of therapy. Diabetic Medicine 1991;8(4):338-345. [PubMed: 1830257]

25. Glasgow RE, Toobert DJ, Hampson S, Brown J, Lewinsohn P, Donnelly J. Improving self-care among older patients with type 11 diabetes: the "Sixty Something" study. Patient Education \& Counseling 1992;19(1):61-74. [PubMed: 1298950]

26. Hendricks LE, Hendricks RT. The effect of diabetes self-management education with frequent followup on the health outcomes of African American men. Diabetes Educator 2000;26(6):995-1002. [PubMed: 11912812]

27. Hernandez C, Hume R, Rodger N. Six-month evaluation of a diabetes self-awareness intervention. Outcomes Management 2003;7:148-158. [PubMed: 14618773]

28. Keyserling TC, Samuel-Hodge CD, Ammerman AS, et al. A randomized trial of an intervention to improve self-care behaviors of African-American women with type 2 diabetes: impact on physical activity. Diabetes Care 2002;25(9):1576-1583. [PubMed: 12196430]

$29 * *$. Klepac, MP. Integrative diabetes education: expansion and evaluation of a holistic program using quantitative and qualitative methodology. The Pennsylvania State University; 2000. p. 330D.Ed.

30. Smith S, Bury G, O'Leary M, et al. The North Dublin randomized controlled trial of structured diabetes shared care. Fam Pract 2004;21(1):39-45. [PubMed: 14760042]

31. Toobert DJ, Glasgow RE, Strycker LA, et al. Biologic and quality-of-life outcomes from the Mediterranean Lifestyle Program: a randomized clinical trial. Diabetes Care 2003 Aug;26(8):228893. [PubMed: 12882850]

32. Trento M, Passera P, Bajardi M, et al. Lifestyle intervention by group care prevents deterioration of type 11 diabetes: a 4-year randomized controlled clinical trial. Diabetologia 2002;45(9):1231-1239. [PubMed: 12242455]

33. Tucker RM, May C, Bennett R, Hymer J, McHaney B. A gym-based wellness challenge for people with type 2 diabetes: effect on weight loss, body composition, and glycemic control. Diabetes Spectr 2004;17(3):176-180.

34. Weinberger M, Kirkman M, Sansa G, et al. A nurse-coordinated intervention for primary care patients with non-insulin-dependent diabetes: impact on glycemic control and health-related quality of life. Journal of General Internal Medicine 1995;10:59-66. [PubMed: 7730940]

35. Wendel I, Durso SC, Zable B, Loman K, Remsburg RE. Group diabetes patient education: a model for use in a continuing care retirement community. Journal of Gerontological Nursing 2003;29(2): 37-44. [PubMed: 12640863]

36. Whittemore R, Chase S, Mandle CL, Roy SC. The content, integrity, and efficacy of a nurse coaching intervention in type 2 diabetes. The Diabetes Educator 2001;27(6):887-898. [PubMed: 12211928] 
37. Wierenga ME. Life-style modification for weight control to improve diabetes health status. Patient Education \& Counseling 1994;23(1):33-40. [PubMed: 7971538]

38. Snoek FJ. Improving quality of life in diabetes: how effective is education. Patient Education \& Counseling 2003;51:1-3. [PubMed: 12915274]

39. Pibernik-Okanovic M, Prasek M, Poljicanin-Filipovic T, Pavlic-Renar I, Metelko Z. Effects of an empowerment-based psychosocial intervention on quality of life and metabolic control in type 2 diabetic patients. Patient Education and Counseling 2004;52(2):193-199. [PubMed: 15132525]

40. Kuijer RG, de Ridder DT. Discrepancy in illness-related goals and quality of life in chronically ill patients: The role of self-efficacy. Psychology \& Health 2003;18(3):313-330.

41. Li C, Ford E, Mokdad A, Jiles R, Giles W. Clustering of multiple healthy lifestyle habits and healthrelated quality of life among adults with diabetes. Diabetes Care 2007;30:1770-1776. [PubMed: 17456843]

42. Fayers, P.; Machin, D. Quality of Life. 2. West Sussex, England: Wiley and Sons Inc; 2007. 
Table 1

Terms Used to Indicate Diabetes Self-Management Interventions

\begin{tabular}{ll|} 
- & adherence behavior therapy \\
- & clinical trial \\
- & compliance \\
- & evaluation \\
- & evaluation study \\
- & evidenced-based medicine \\
- & health care evaluation \\
- & health behavior \\
- & health education \\
- & health promotion \\
- & intervention \\
- & outcome and process assessment \\
- & patient education program \\
- & program development \\
- & program evaluation \\
- & self-care \\
- & treatment outcome \\
- & validation study \\
- & exercise \\
- & physical activity \\
- & physical fitness \\
- & exertion \\
- & physical education and training \\
\hline
\end{tabular}




\section{Table 2}

Inclusion Criteria for Primary Studies

- Participants 21 years of age or older

- $\quad$ Participants with diagnosed diabetes (either type 1 or type 2 )

- Diabetes self-management intervention which included recommendation to increase physical activity (the vast majority of retrieved diabetes self-management intervention trials included recommendations to increase physical activity)

- QOL measurement post-intervention with sufficient data to calculate an effect size (e.g. means and SD/ $\mathrm{SE}$; $\mathrm{t}$ statistic; exact $\mathrm{p}$ value from $\mathrm{t}$ statistic) 
Table 3

Characteristics of Primary Studies Included in Meta-Analyses

\begin{tabular}{|l|c|c|c|c|}
\hline Characteristic & $\boldsymbol{k}$ & Minimum & Mean (SD) & Maximum \\
\hline Mean age (years) & 18 & 44.50 & $59.08(8.00)$ & 79.00 \\
\hline Sample size per study & 20 & 8.00 & $104.75(120.16)$ & 386.00 \\
\hline Number subjects- treatment group & 20 & 8.00 & $65.45(60.48)$ & 240.00 \\
\hline Number of control group subjects & 8 & 23 & $98.25(69.53)$ & 203 \\
\hline Proportion attrition-treatment group & 18 & 0 & $.16(.13)$ & .50 \\
\hline Proportion attrition-control group & 7 & 0 & $.12(.12)$ & .35 \\
\hline Baseline HbA1c & 14 & 7.05 & $8.38(1.59)$ & 11.30 \\
\hline Baseline body mass index & 7 & 28.67 & $33.34(3.29)$ & 36.50 \\
\hline Percent women & 17 & 0 & $55(37)$ & 100 \\
\hline Number educ./motivation sessions & 18 & 2 & $7.67(5.46)$ & 24 \\
\hline Min/session education/motivation & 13 & 15 & $117.69(79.57)$ & 300 \\
\hline Weeks over intervention delivered & 18 & 1 & $27.56(46.71)$ & 208 \\
\hline
\end{tabular}




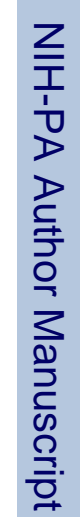

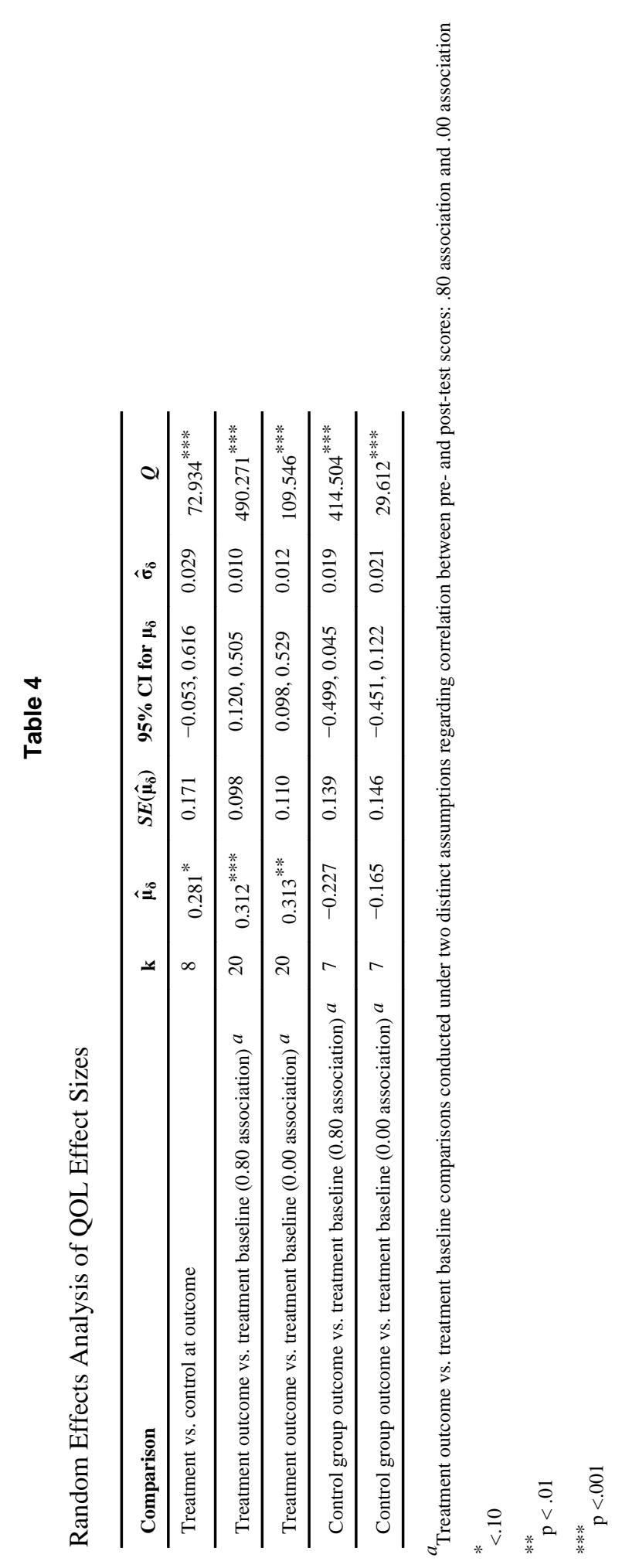

Diabetes Educ. Author manuscript; available in PMC 2010 February 16. 